\title{
EXPORT BALANCING METHOD OF PRODUCTION-CONSUMPTION MODEL
}

\author{
Ewa Ladyga, Marek Ladyga \\ Institute of Mathematics, Czestochowa University of Technology \\ Częstochowa, Poland \\ ewa.ladyga@im.pcz.pl,marek.ladyga@im.pcz.pl \\ Received: 15 October 2016; accepted: 15 November 2016
}

\begin{abstract}
This paper presents an export balancing method of the production-consumption model. All contractors have their share in balancing the model, and the compromise they reach is determined explicitly and precisely according to the requirements of decisionmakers.
\end{abstract}

Keywords: export balancing method, unsustainable production-consumption model

\section{Introduction}

In every society there are producers, who must produce something to make a profit and consumers, who must buy something to meet their needs. When the whole production is absorbed by the market, and producers are not interested in expanding it, then we have the so called natural balance [1]. Because of the fact that the Polish economy is limited due to supplies, conflicts arise between producers and consumers quite often. The issue of conflicting goals of producers (increase in production) and consumers (decrease in demand) is shown in [2]. The method of mutual compromise between contractors to balance demand and supply consists in:

a) determining benchmarks and their scopes, which will allow one to reach a compromise in the unsustainable production-consumption model, where the demand for particular goods exceeds their supply,

b) determining mutual relationships between particular contractors and benchmarks,

c) evaluating the scope of compromise in case of all contractors on the basis of the above-mentioned data.

This paper presents the unsustainable production-consumption model, in which contractors, including producers and consumers of particular goods, play a part. 


\section{The solution of the problem}

In this model it is assumed that the total supply of goods does not cover the total demand for them in a given period of time, which can be shown in the form of the - mentioned demand and supply vector fulfilling the following conditions:

1) $\forall i: i \leq m \quad p_{i}>0$,

2) $\forall i: m+1 \leq i \leq n \quad p_{i}<0$,

3) $\sum_{i=1}^{n} p_{i}<0, \quad \vec{p}=\left(p_{1}, p_{2}, \ldots, p_{n}\right)$.

The first coordinates (from 1 to $m$ ) of the vector $\vec{p}$, denoted by positive numbers represent producers supply. The final coordinates $n-m$ of the vector $\vec{p}$ denoted by negative numbers represent consumers demand. Condition no. 3 shows that the production does not cover the demand for particular goods. To balance the model (when supply equals demand) contractors must make concessions. The possible maximum concessions are denoted by the vector fulfilling $\vec{u}=\left(u_{1}, u_{2}, \ldots, u_{n}\right)$ the following conditions:

1) $\forall i \leq n \quad u_{i} \geq 0$,

2) $\sum_{i=1}^{n}\left(p_{i}+u_{i}\right) \geq 0$.

For $i \leq m u_{i}$ means a maximum possible increase in production of this producer. For $i>m u_{i}$ means a maximum possible decline in demand of this consumer. Condition no. 2 makes balancing possible, as the total possible number of concessions of all the contractors is smaller than the number needed to balance the model.

The question is which contractor should verify their demand or supply and to what extent.

Let's assume that KR is not an empty set of benchmarks (the power sets KR is equal) on the basis of which it is decided which contractor should verify their demand or supply and to what extent. The KR set may include both minimum and maximum benchmarks as well as conditions imposed by contractors. To find out to what extent all contractors meet particular benchmarks, the following mapping must be used:

$$
f: K \times K R \rightarrow R+\cup\{0\}
$$

the $\forall i \leq n \quad \exists_{j} \leq k$, one that $f(i, j) \neq 0$.

The real number indicates $f(i, j)$ to what extent the i-contractor meets $\mathrm{j}$-benchmarks. It is convenient to present the mapping values $f$ in the form of a matrix 
$F=\left[\begin{array}{ccc}f(1,1), & \ldots, & f(1, k) \\ \vdots & & \vdots \\ f(n, 1), & \ldots, & f(n, k)\end{array}\right]$

$J$-column of the matrix represents how all contractors behave towards $j$-benchmark. By contrast, $i$-row shows to what extent a given $j$-contractor meets particular benchmarks. So as to compare the values for various $f(i, j)$ contractors and various benchmarks the matrix $F$ must be normalized. Let the vector $\vec{w}=\left(w_{1}, w_{2}, \ldots, w_{k}\right)$ be a vector of particular benchmark weights, meaning for each one $i \leq k \quad w_{i} \geq 0$ and $\left(\vec{w}, \overrightarrow{e_{k}}\right)=1$, where $\overrightarrow{e_{k}}=(1,1, \ldots, 1),\left(\vec{w}, \overrightarrow{e_{k}}\right)$ is the scalar product of $\vec{w}$ and $\overrightarrow{e_{k}}$ vectors. Taking into account the above-mentioned requirements, it is necessary to use the matrix coupled with $f$ mapping, the matrix is denoted by, $\mathrm{A}(f)=\left[a_{i j}\right]_{n \times k}$ where $a_{i j}=\frac{w_{j} f(i, j)}{\sum_{t=1}^{n} f(t, j)}$.

Aggregation of $\mathrm{A}(f)$-row of the matrix allows for evaluation of $i$-contractor's attitude towards all benchmarks. Hence, there is a vector $\vec{a}=\left(a_{1}, \ldots, a_{n}\right)=\mathrm{A}(f) \overrightarrow{e_{k}^{T}}$ introduced, which is of vital importance while determining concessions of contractors. This vector has been called the price of contractors concessions.

Having assigned three vectors:

a) demand-supply

$$
\vec{p}=\left(p_{1}, p_{2}, \ldots, p_{n}\right), \text { such as that }\left(\vec{p}, \overrightarrow{e_{n}}\right)<0
$$

b) maximum concession

$$
\vec{u}=\left(u_{1}, u_{2}, \ldots, u_{n}\right) \text {, such as that }\left(\overrightarrow{p+u}, \vec{e}_{n}\right)>0
$$

c) the price of contractors' concessions with regard to benchmarks

$$
\vec{a}=\left(a_{1}, a_{2}, \ldots, a_{n}\right), \text { such as that } \vec{a}=\mathrm{A}(f) \cdot \overrightarrow{e_{k}^{T}}
$$


and assuming:

$$
\overrightarrow{p^{\mathrm{o}}}=\vec{p}, \overrightarrow{a^{\mathrm{o}}}=\vec{a}, \mathrm{~N}^{\mathrm{o}}=\{1,2, \ldots, n\}
$$

for $l \geq 1$ we define the following values:

$$
\begin{gathered}
\gamma^{l}=-\left(\overrightarrow{p^{l-1}, \overrightarrow{e_{n}}}\right) \\
\mathrm{N}^{l}=\left\{i \in \mathrm{N}^{\circ}: \sum_{j=1}^{l} a_{i}^{j-1} r^{j} \leq u_{i}\right\} \\
\overrightarrow{a^{l}}=\left(a_{1}^{l}, a_{2}^{l}, \ldots, a_{n}^{l}\right)
\end{gathered}
$$

where

$$
\begin{gathered}
a_{i}^{l}= \begin{cases}0 & \text { for } \quad i \notin \mathrm{N}^{l} \\
\frac{a_{i}^{l-1}}{\sum_{j \in \mathrm{N}^{l-1}} a_{j}^{l-1}} \text { for } \quad i \in \mathrm{N}^{l}\end{cases} \\
\overline{\mathrm{N}}^{l}=\mathrm{N}^{l-1} \backslash N^{l} \\
\vec{p}^{l}=\left(p_{1}^{l}, \ldots, p_{n}^{l}\right)
\end{gathered}
$$

where

$$
p_{i}^{l}=\left\{\begin{array}{lll}
p_{i}^{l-1}+r^{l} a_{i}^{l-1} & \text { for } & i \in \mathrm{N}^{l} \\
p_{i}+u_{i} & \text { for } & i \in \overline{\mathrm{N}}^{l} \\
p_{i}^{l-1} & \text { for } & i \in \bigcup_{j<l} \bar{N}^{j}
\end{array}\right.
$$

The iterative process (2)-(10), the properties of which are discussed in [3], is finished for such a $s$-iteration that $r^{s}=0$.

In the presented algorithm, it is assumed that users are acquainted with the mechanism responsible for economic phenomena and they generally know what elements the set of benchmarks KR should contain and what values should be assigned $f(i, j)$ to mapping. The problem of the explicit indication of vector $\overrightarrow{p^{l}}$ is presented in the following statement. 
If:

a) $\vec{a}=\left(a_{1}, \ldots, a_{n}\right), \vec{b}=\left(b_{1}, \ldots, b_{n}\right)$ the price of contractors concessions with regard to benchmarks,

b) vectors $\overrightarrow{p^{l}}$ i $\overrightarrow{q^{l}}$ are 1-fold $\vec{p}$ iterations of the vector which are achieved by means of vectors $\vec{u}, \overrightarrow{\mathrm{a}}$ and $\vec{u}, \vec{b}$ then $p_{i}^{l}=q_{i}^{l} \Leftrightarrow a_{i}^{l-1}=b_{i}^{l-1}$.

According to this statement, the vector of demand and supply in each iteration, that is to say the vector which balances the model, is indicated accurately to the price of contractors concessions with regard to benchmarks. Unfortunately, because of insufficient knowledge, the explicit indication of the set KR, $f$ mapping values, (1), and by extension the matrix coupled with $\mathrm{f}$ mapping, is not always possible. It happens sometimes that the knowledge on the mechanism of economic phenomena which are to be explored is so limited that all one can do is try to stick to a list of elements which are more or less adequate to the set KR $[4,5]$. The same situation may occur in the case of indication of $f$ mapping values. The question appears which elements out of the ones from the set KR should be taken into account, and which ones should be rejected, as taking all of them into account could aggravate the problem and blear their real scopes. In my opinion, in such cases, experts opinions should be considered. If a given team is well-chosen, that is to say there are very reliable experts who specialize in problematic aspects of the analysed phenomenon and its circumstances, then as a result of consultation and compilation of their opinions, it will be possible to decide which elements should be included in the set KR and what values should be assigned to $f$ mapping. However, if the experts represent different types of corporations, their opinions may differ [4]. Then, instead of one matrix coupled with the adjusting mapping one will get a few different variants.

Let's assume that $\overrightarrow{V^{i}}$ means $i$-vector, which balances the production and consumption model achieved by means of iteration (2)-(10) on the basis of $i$ matrix coupled with $f$ mapping. While $m\left(\overrightarrow{V^{i}}\right)$ means a real number (not a negative one) which is matched with the vector $\overrightarrow{V^{i}}$. This number is called a vector usability measure $\vec{V}^{i}$ for $i=1,2, \ldots, t$.

Since numbering of vectors is arbitrary, one can assume that $m\left(\overrightarrow{V^{1}}\right), m\left(\overrightarrow{V^{2}}\right), \ldots m\left(\overrightarrow{V^{t}}\right)$ the sequence is non-decreasing, where $t$ is a number of matrices coupled with $\mathrm{f}$ mapping (1). The alternative solution is a solution in which users choose one of the vectors from the specialized $U$ set. The alternative 
solution can work out only when it is acceptable in reality [5]. That is why the following rule of communication of the $U$ set is applied.

$U=\left\{\overrightarrow{V^{i}}: \mathrm{m}\left(\overrightarrow{V^{1}}\right)-\mathrm{m}\left(\vec{V}^{i}\right) \leq a\right\}$, where $a$ is a real number determined in advance and belonging to the range $\langle 0,1\rangle$.

The question how to choose a number $m\left(\overrightarrow{V^{i}}\right)$ must still be answered. I would suggest using an export method. Mapping does $f(1)$ not need to be explicitly determined. Thus, one can get different variants of A matrix coupled with $f$ mapping. It is assumed that there are $t$ of such matrices $A^{1}, A^{2}, \ldots, A^{t}$. As a result one can get solutions $\overrightarrow{V^{1}}, \overrightarrow{V^{2}}, \ldots, \overrightarrow{V^{t}}$ where $\vec{V}^{i}=\left(V_{1}^{i}, V_{2}^{i}, \ldots, V_{n}^{i}\right), n$ means a number of contractors, $i=1,2, \ldots, t$. Using a group of experts, each solution may be assigned to some measure of meaningfulness $m\left(\overrightarrow{V^{i}}\right)$. It is assumed that this group amounts to $\mathrm{w} \geq 1$ people. Each expert assigns each solution some non-negative number $m\left(V^{i j}\right)$ for $i \leq t, j \leq w$. The number $m\left(V^{i j}\right)$ defines the measure of meaningfulness of $i$-solution carried out by $j$-expert. It must be noted that number sequences $m\left(V^{11}\right), \ldots, m\left(V^{1 w}\right), m\left(V^{21}\right), \ldots, m\left(V^{2 w}\right)$, $\ldots, m\left(V^{t 1}\right), \ldots, m\left(V^{t w}\right)$ should be sequences of the same rows. Otherwise, some number sequences for $m\left(V^{i 1}\right), \ldots, m\left(V^{i q}\right)$ must be normalized so that they could match sequences rows $m\left(V^{j 1}\right), \ldots, m\left(V^{j q}\right)$ for $i \neq j, q \leq w$. Having assigned values $m\left(V^{i j}\right)$ for $i \leq t, j \leq m$, one should calculate the initial measure of $i$-solution, as an arithmetic mean of $i$-solution carried out by all the experts. If the initial measure of $i$-solution is denoted by $\bar{m}\left(V^{i}\right)$ :

$\bar{m}\left(\overrightarrow{V^{i}}\right)=\frac{1}{w} \sum_{j=1}^{w} m\left(V^{i j}\right)$ for $i \leq t$

Normalizing these initial measures to unit one gets:

$m\left(\overrightarrow{V^{i}}\right)=\frac{\bar{m}\left(\overrightarrow{V^{i}}\right)}{\sum_{j=1}^{w} \bar{m}\left(\overrightarrow{V^{j}}\right)}$ for $i \leq t$. 
The solutions $\overrightarrow{V^{1}}, \overrightarrow{V^{2}}, \ldots, \overrightarrow{V^{t}}$ are arranged in non-increasing sequence according to the measures $m\left(\overrightarrow{V^{i}}\right)$.

Let $m\left(\overrightarrow{V^{1}}\right) \geq m\left(\overrightarrow{V^{2}}\right) \geq \ldots \geq m\left(\overrightarrow{V^{t}}\right)$.

The $U$ set contains only these vectors $\vec{V}^{i}$, the measure $m\left(\overrightarrow{V^{i}}\right)$ of which differs from the solution having the biggest measure by no more than $a$. I suggest the number $a$ is a percent of difference between $m\left(\overrightarrow{V^{1}}\right)$ and $m\left(\overrightarrow{V^{t}}\right)$. The percent is determined by the algorithm user. If $a$ is smaller than the difference $m\left(\overrightarrow{V^{1}}\right)-m\left(\overrightarrow{V^{2}}\right)$, the $U$ set contains only $\vec{V}^{1}$ solution. If $a$ is bigger than the difference $m\left(\overrightarrow{V^{1}}\right)-m\left(\overrightarrow{V^{2}}\right)$, the $U$ set contains all the solutions: $\overrightarrow{V^{1}}, \overrightarrow{V^{2}}, \ldots, \overrightarrow{V^{t}}$. Thus, the conclusion is that for each $a \in\langle 0,1\rangle$ the $U$ set of alternative solutions differs from an empty set.

\section{Conclusion}

In this paper we showed that:

a) it is possible to consider the problem of conflictual objectives on the line producer (increase in supply) - consumer (decrease in demand) using the mathematical economic model,

b) all contractors are responsible for balancing the unsustainable productionconsumption model,

c) the compromise is achieved explicitly and accurately with regard to the decision-makers' requirements.

\section{References}

[1] Hellwig Ż., Równowaga ekonomiczna, Pojęcie - kontrowersje, Ekonomista 1981, 3/4.

[2] Ladyga M., Tkacz M., The properties of method balancing the unsustainable production and consumption model, Scientific Research of the Institute of Mathematics and Computer Science 2012, 3(11), 105-109. 
[3] Ladyga M., Lovasova R., The method of balancing the production and consumption model in the case of indivisible goods, Polish Journal of Management Studies 2015, 11(2), 83-90.

[4] Dima I.C., Grabara J., Kolcun M., Econometric model for planning the industrial production, Journal of Applied Mathematics and Computational Mechanics 2013, 12(1), 11-20.

[5] Brzeziński S., Grabara J., Kot S., Outsourcing performance in the power plants, [in:] Elektroenergetika 2015, Proceedings of the 8th International Scientific Symposium on Electrical Power Engineering, September 16-18, 2015, Stara Lesna, Slovak Republic 2015, 28-30. 